\title{
GENERALIZATION OF THE WHITNEY-MAHOWALD THEOREM
}

\author{
BANG-HE LI
}

\begin{abstract}
The Whitney-Mahowald theorem gave normal Euler number $(\bmod 4)$ for embeddings of a closed $2 n$-manifold in Euclidean $4 n$-space. We generalize this theorem to embeddings of closed $2 n$-manifolds in an oriented $4 n$-manifold with an approach in the framework of unoriented bordism groups of maps.
\end{abstract}

\section{INTRODUCTION}

Given a map $f: M \rightarrow N$, where $M$ and $N$ are smooth connected manifolds with dimensions $n$ and $2 n$ respectively, and $M$ is closed, $N$ is oriented, there is a question:

What is the set of the normal Euler classes of smooth embeddings in the homotopy class $[f]$ ?

An orientation of $N$ and a map $f: M \rightarrow N$ determine an isomorphism $H^{n}(M, \widetilde{Z}) \rightarrow Z$ which sends the normal Euler class of an embedding $g$ in $[f]$ to an integer $\chi(g)$ called the normal Euler number $g$, where $\tilde{Z}$ is the local integer coefficients associated to the orientation line bundle of $M$. For odd $n$, the normal Euler classes are always zero. Hence Question $(*)$ is interesting only for even $n$.

H. Whitney [Wh1] first proved that any $n$ manifold embeds in $R^{2 n}$. By using Whitney's technique, J. Milnor proved in [Mi] that if $N$ is simply connected and $n>2$, then any $f: M \rightarrow N$ is homotopic to embeddings. The following more general result is due to Haefliger [Ha]:

Theorem 1 (Haefliger). If $n>2$ and $f: M^{n} \rightarrow N^{2 n}$ is a map with $f_{*}$ : $\pi_{1}(M) \rightarrow \pi_{1}(N)$ surjective, then $f$ is homotopic to embeddings.

If $M$ is orientable, then the normal Euler numbers of embeddings are uniquely determined by their homotopy classes. However, if $M$ is nonorientable, the situation changes. Whitney [Wh2] in the case $n=2$, and Mahowald [Mah] in the case of $n$ even, proved that if $f: M^{n} \rightarrow R^{2 n}$ is an embedding, then

$$
\chi(f)=2 \bar{w}_{1}(M) \bar{w}_{n-1}(M) \bmod 4,
$$

where $2 \bar{w}_{1}(M) \bar{w}_{n-1}(M)$ is understood as the image of the dual Stiefel-Whitney number $\bar{w}_{1}(M) \bar{w}_{n-1}(M)$ under the natural inclusion $Z_{2} \rightarrow Z_{4}$.

Received by the editors September 2, 1993.

1991 Mathematics Subject Classification. Primary 57N35.

This work is partially supported by the National Funds of Science of P. R. China. 
Malyi [Mal] proved that if $n>2$ is even and $M^{n}$ is nonorientable, then for any integer $x$ with $x=2 \bar{w}_{1}(M) \bar{w}_{n-1}(M) \bmod 4$, there is an embedding $f: M^{n} \rightarrow R^{2 n}$ with $\chi(f)=x$.

W. S. Massey gave a new proof of Mahowald's theorem in [Mas], by using the following formula proved also by him:

$$
\widetilde{P}\left(U_{2}\right)=\left(\tilde{\rho}_{4}\left(X_{n}\right)+\tilde{\theta}\left(w_{1} w_{n-1}\right)\right) \cdot U,
$$

where $U$ is the Thom class of an $n$-dimensional vector bundle $\xi$ over $B$ with $n$ even, $X_{n}$ the Euler class of $\xi$, both $U$ and $X_{n}$ take local integer coefficients $\widetilde{Z}$ determined by $\xi, U_{2}=U \bmod 2, w_{i}$ the $i$ th Whitney class of $\xi, \widetilde{P}$ the Pontryagin square, and

$$
\tilde{\rho}: H^{q}(B, \widetilde{Z}) \rightarrow H^{q}\left(B, \widetilde{Z}_{4}\right), \quad \tilde{\theta}: H^{q}\left(B, Z_{2}\right) \rightarrow H^{q}\left(B, \widetilde{Z}_{4}\right)
$$

the natural homomorphisms.

To generalize Mahowald's theorem to embeddings of $M^{n}$ in an oriented $N^{2 n}$, we define first $P: H_{n}\left(N, Z_{2}\right) \rightarrow Z_{4}$ as follows:

For any $x \in H_{n}\left(N, Z_{2}\right)$, take a compact submanifold $N_{x}^{2 n}$ of $N$ so that $x=i_{*} y$, where

$$
i_{*}: H_{n}\left(N_{x}, Z_{2}\right) \rightarrow H_{n}\left(N, Z_{2}\right)
$$

is the natural homomorphism and $y \in H_{n}\left(N_{x}, Z_{2}\right)$. Let

$$
D y \in H^{n}\left(N_{x}, \partial N_{x}, Z_{2}\right) \cong H^{n}\left(N_{x} / \partial N_{x}, Z_{2}\right)
$$

be the Lefschetz dual of $y$. Then

$$
P(x)=\left\langle\widetilde{P}(D y),\left[N_{x} / \partial N_{x}\right]\right\rangle,
$$

where $\left[N_{x} / \partial N_{x}\right]$ is the fundamental class of

$$
H_{2 n}\left(N_{x} / \partial N_{x}, Z_{4}\right) \cong Z_{4}
$$

determined by the orientation of $N_{x}$ inherited from that of $N$, and $\langle$, stands for the Kronecker product.

It is easy to see that $P$ is well defined and if $n$ is even, then

$$
P(x+y)=P(x)+P(y)+2 x \cdot y,
$$

where $x \cdot y$ is the intersection number (the proof depends on a formula for the Pontryagin square; cf. [MT, p. 21]).

Now we are in a position to state

Theorem 2. Let $n$ be even.

(1) If $f: M^{n} \rightarrow N^{2 n}$ is an embedding, then

$$
\chi(f)=P\left(f_{*}[M]\right)+2 w_{1}(f) w_{n-1}(f) \bmod 4
$$

where $[M]$ is the generator of $H_{n}\left(M, Z_{2}\right) \cong Z_{2}$, and $w_{i}(f)$ the ith normal Whitney class of $f$.

(2) If $n>2, M$ is nonorientable, and $f$ is a map with $f_{*}: \tilde{\pi}_{1}(M) \rightarrow \pi_{1}(N)$ surjective, where $\tilde{\pi}_{1}(M)$ is the subgroup of $\pi_{1}(M)$ consisting of orientationpreserving elements, then normal Euler numbers of embeddings in $[f]$ fill the $\bmod 4$ residue class $P\left(f_{*}[M]\right)+2 w_{1}(f) w_{n-1}(f)$.

Corollary 1. If $n$ is even, and $f, g: M^{n} \rightarrow N^{2 n}$ are homotopic embeddings, then $\chi(f)=\chi(g) \bmod 4$. 
Corollary 2 (The generalized Whitney congruence of Rohlin [Ro]). Let $f$ : $M^{2} \rightarrow N^{4}$ be an embedding, where $N$ is oriented closed and $f_{*}[M]$ is characteristic; then

$$
\sigma(N)=\chi(f)+2 \chi(M) \bmod 4,
$$

where $\sigma(N)$ is the signature of $N$, and $\chi(M)$ stands for the Euler characteristic number of $M$.

Theorem 3. Let $\mathscr{N}_{n}(N)$ be the bordism group of maps from closed (possibly nonorientable.) $n$-manifolds into the oriented $2 n$-manifold $N$. Then $P\left(f_{*}[M]\right)+$ $2 w_{1}(f) w_{n-1}(f)$ gives a map $q: \mathscr{N}_{n}(N) \rightarrow Z_{4}$ with the following properties:

(1) Any element $x \in \mathscr{N}_{n}(N)$ includes embeddings with normal Euler numbers mod 4 equal to $q(x)$.

(2) A self-transversal immersion with only double points in $x$ has mod 4 normal Euler number equal to $q(x)$ (or $q(x)+2$ ) if and only if its number of self-intersection points is even (or odd).

(3) Let $x, y \in \mathscr{N}_{n}(N)$ be represented by $f: M_{1} \rightarrow N$ and $g: M_{2} \rightarrow N$, and define $x \cdot y$ as $f_{*}\left[M_{1}\right] \cdot g_{*}\left[M_{2}\right] \in Z_{2}$. Then $x \cdot y$ gives a bilinear form on $\mathscr{N}_{n}(N)$ and

$$
q(x+y)=q(x)+q(y)+2 x \cdot y .
$$

In general, it is difficult to calculate Pontryagin squares. We shall give another formula for $q(x)$ in a special case that $N$ is the total space of the orientation line bundle of a manifold $K^{2 n-1}$, e.g. $N=R^{2 n-1} \times R=R^{2 n}$.

Let $f: M^{n} \rightarrow K^{2 n-1}$ be a map, $K^{\prime}$ a compact $(2 n-1)$-dimensional submanifold of $K$ containing $f(M)$, and $D: H_{n}\left(K^{\prime}, Z_{2}\right) \rightarrow H^{n-1}\left(K^{\prime}, \partial K^{\prime} ; Z_{2}\right)$ the Lefschetz dual. Then

$$
u(f)=f^{*} D f_{*}[M] \in H^{n-1}\left(M, Z_{2}\right)
$$

is well defined. We have

Theorem 4. If $N$ is the total space of the orientation line bundle of $K^{2 n-1}$ with $n$ even, and $f: M \rightarrow N$ is an embedding, then

$$
\chi(f)=2 \tilde{w}_{1}(f)\left(u(f)+\tilde{w}_{n-1}(f)\right) \bmod 4,
$$

where $\tilde{w}_{i}(f)$ is the ith stable normal Whitney class of $f$ regarded as a map $M \rightarrow K$, and $u(f)$ is understood similarly.

Remark 1. The proof of Theorem 4 is geometric, hence different from those of Mahowald and of Massey for $N=R^{2 n}$.

Remark 2. By Theorem 3 and the proof of Theorem 4, we have

$$
q(x)=2 \tilde{w}_{1}(f)\left(u(f)+\tilde{w}_{n-1}(f)\right),
$$

where $f \in x \in \mathscr{N}_{n}(K)=\mathscr{N}_{n}(N)$, and a formula expressing $P\left(f_{*}[M]\right)$ in terms of $u(f), w_{1}(K)$, and $w_{i}(f), i \leq n-1$, can be gotten. From this we see that if $K^{2 n-1}$ is orientable with $n$ even and $N=K \times R, y \in H_{n}\left(N, Z_{2}\right)$ with $P(y) \neq 0$, then there is no map $f: M^{n} \rightarrow N$ with $M$ orientable and $f_{*}[M]=y$. 
Example. Let $g: M=R P^{n} \# R P^{n} \rightarrow R P^{n}$ be the map collapsing the second copy to a point, and $f$ be the composition

$$
M \stackrel{g}{\rightarrow} R P^{n} \subset R P^{2 n-1} \subset R P^{2 n-1} \times R .
$$

Then

$$
f_{*}: \tilde{\pi}_{1}(M) \rightarrow \pi_{1}\left(R P^{2 n-1} \times R\right)
$$

is surjective, and it follows from (2) of Theorem 2 that if $n>2$ is even, then the normal Euler numbers of the embeddings homotopic to $f$ fill a mod 4 residue class. Now Theorem 4 tells us that this class is $4 Z$. And we see that for the generator $x$ of $H_{n}\left(R P_{2 n-1} \times R\right), P(x)=2$, and hence $x$ is not represented by maps from orientable $n$-manifolds.

Remark 3. The earliest preprint of this paper was typed in 1989, under the title "Embedding $n$-manifolds in $2 n$-manifolds" and was used and quoted in [Li3]. The main content of the paper was given in a talk in a conference held at Tokyo University, September, 1990.

Remark 4. Recently, Yamada [Ya] found the formula in part (1) of Theorem 2 for the case $n=2$ with $H_{1}(M, Z)=0$ independently, using a geometric method.

\section{Proof of Theorem 2}

Let $f: M \rightarrow N$ be an embedding, $N_{f}$ a compact tubular neighbourhood of $f(M)$. Regard $N_{f}$ as the disk bundle of the normal bundle of $f$; we have by Massey's formula

$$
\widetilde{P}\left(U_{2}\right)=\left(\tilde{\rho}_{4}(X(f))+\theta\left(w_{1}(f) w_{n-1}(f)\right)\right) \cdot U
$$

where $X(f)$ is the normal Euler class.

Let $\left[N_{f} / \partial N_{f}\right]$ be the fundamental class of

$$
H^{2 n}\left(N_{f} / \partial N_{f}, Z_{4}\right) \cong Z_{4}
$$

corresponding to the orientation of $N_{f}$ inherited from that of $N$. Since $U_{2} \in$ $H^{n}\left(N_{f}, \partial N_{f} ; Z_{2}\right)$ is the Lefschetz dual of $f_{*}[M] \in H_{n}\left(N_{f}, Z_{2}\right)$, we have

$$
\widetilde{P}\left(U_{2}\right)=P\left(f_{*}[M]\right)\left[N_{f} / \partial N_{f}\right] .
$$

Let $\chi(f)$ be the normal Euler number determined by the orientation of $N$. Then the Thom isomorphism

$$
H^{n}\left(M, \widetilde{Z}_{4}\right) \cong H^{2 n}\left(N_{f} / \partial N_{f}, Z_{4}\right)
$$

given by $x \rightarrow x \cdot U$ sends

$$
\left(\tilde{\rho}_{4}(X(f))+\tilde{\theta}\left(w_{1}(f) w_{n-1}(f)\right)\right) \cdot U
$$

to

$$
\left(\chi(f)+2 w_{1}(f) w_{n-1}(f)\right)\left[N_{f} / \partial N_{f}\right]
$$

hence

$$
\chi(f)=P\left(f_{*}[M]\right)+2 w_{1}(f) w_{n-1}(f) \bmod 4
$$

and (1) is proved.

Suppose $n>2$ and $f_{*}: \tilde{\pi}_{1}(M) \rightarrow \pi_{1}(N)$ is surjective. Then by Theorem $1, f$ is homotopic to embeddings and we may assume $f$ is an embedding. 
For any $m \in Z$, take a self-transversal immersion $h: S^{n} \rightarrow S^{2 n}$ with $2|m|$ self-intersection points and $\chi(h)=4 \mathrm{~m}$. Making a suitable connected sum of $f$ and $h$, we get a self-transversal immersion $g=f \# h: M \rightarrow N$ homotopic to $f$ with $2|m|$ self-intersection points and $\chi(g)=\chi(f)+4 m$. Assume $a_{1}, b_{1}, a_{2}, b_{2}, \ldots, a_{2|m|}, b_{2|m|}$ are distinct points in $M$ such that $g\left(a_{i}\right)=$ $g\left(b_{i}\right)$.

Choose simple curves $I_{i}, i=1,2$, connecting $a_{i}$ and $b_{i}$ such that $I_{1} \cap I_{2}=$ $\varnothing$ and $I_{i} \cap\left\{a_{3}, b_{3}, \ldots, a_{2|m|}, b_{2|m|}\right\}=\varnothing$. Then $g\left(I_{1}\right)$ and $g\left(I_{2}\right)$ form a simple closed curve $\gamma$ in $N$. Given orientations of $T(M)$ on $I_{1}$ and $I_{2}$, the signs of self-intersections of $g$ at $g\left(a_{1}\right)$ and $g\left(a_{2}\right)$ are determined. The surjectivity of $f_{*}: \tilde{\pi}_{1}(M) \rightarrow \pi_{1}(N)$ together with its implicate that there exist elements in $\pi(M) \backslash \tilde{\pi}(M)$ which are in the kernel of $f_{*}$ allows us to choose $I_{2}$ so that $\gamma$ is nullhomotopic and the signs at $g\left(a_{1}\right)$ and $g\left(a_{2}\right)$ are opposite. Thus, by Whitney's technique, we can get an immersion regularly homotopic to $g$ with $2|m|-2$ self-intersection points. Continuing in this way, we will get at last an embedding regularly homotopic to $g$. This proves (2).

Proof of Corollary 2. By a formula of $\mathrm{Wu}$ (see [Wu] or [Th]),

$$
P\left(f_{*}[M]\right)=\widetilde{P}\left(w_{2}(N)\right)=p_{1}(N) \bmod 4+2 w_{4}(N),
$$

where $p_{1}$ is the first Pontryagin class of $N . w_{4}(N)+\sigma(N) \bmod 2$ is an $\mathscr{N}_{4}^{\text {so }}$ invariant, and $w_{4}\left(C P^{2}\right)+\sigma\left(C P^{2}\right) \bmod 2=0$; hence $w_{4}(N)+\sigma(N) \bmod 2=$ 0 for any $N$. This fact together with $p_{1}(N)=3 \sigma(N)$ implies $P\left(f_{*}[M]\right)=$ $\sigma(N) \bmod 4$. Now, Corollary 2 follows from (1) of Theorem 2.

\section{Proof of Theorem 3}

First, since $f_{*}([M])$ and $w_{1}(f) w_{n-1}(f)$ are bordism invariants, $q$ is well defined.

Now, let $f: M \rightarrow N$ be a self-transversal immersion, and $b \in N$ be a self-intersection point of $f$ such that $b=f\left(a_{1}\right)=f\left(a_{2}\right)$, but $a_{1} \neq a_{2}$. Take a neighbourhood of $b$ which corresponds to $R^{2 n}$ diffeomorphically such that the image of $f$ in this neighbourhood corresponds to $R^{n} \times 0 \cup 0 \times R^{n}$. For $x \in R^{n}$ with $|x|<2$, let $v(x, 0)=(0, x), v(0, x)=(x, 0)$. Then $v$ extends to a normal vector field of $f$ denoted by $v$ also. We may assume $v$ is transversal to the zero section since it is already so at $a_{1}$ and $a_{2}$. The normal Euler number of $f$ is the algebraic sum of the zeros of $v$, and the total contribution of $a_{1}$ and $a_{2}$ is \pm 2 .

Let $\gamma$ be a curve in the plane as shown in Figure 1.

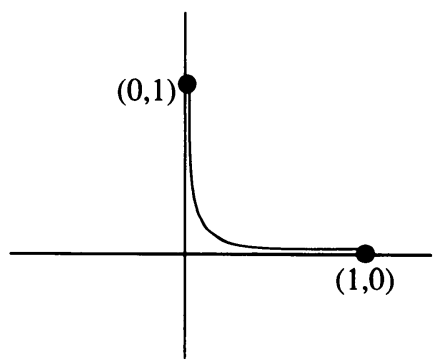

FIGURE 1 


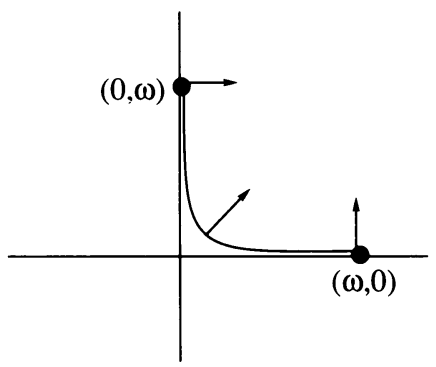

FIGURE 2

We assume $\gamma(t)=\left(\gamma_{1}(t), \gamma_{2}(t)\right), 0 \leq t \leq 1$, such that $\gamma(0)=(0,1), \gamma(1)=$ $(1,0)$, and $\gamma$ contacts with the $x$-axis and $y$-axis smoothly.

Let

$$
\begin{aligned}
D^{n} & =\left\{x \in R^{n}|| x \mid<1\right\}, \\
S^{n-1} & =\left\{x \in R^{n}|| x \mid=1\right\} .
\end{aligned}
$$

Take off the neighbourhoods of $a_{1}$ and $a_{2}$ in $M$ which correspond to $D^{n} \times 0$ and $0 \times D^{n}$, and then add $S^{2 n-1} \times I$ naturally; we get a new manifold $M^{\prime}$. Mapping $S^{n-1} \times I$ to $R^{2 n}$ by

$$
(\omega, t) \rightarrow\left(\gamma_{1}(t) \omega, \gamma_{2}(t) \omega\right),
$$

we have an immersion $f^{\prime}$ of $M^{\prime}$ in $N$ and a normal vector field $\nu^{\prime}$ of $f^{\prime}$ which are identical with $f$ and $v$ respectively outside the neighbourhoods of $a_{1}$ and $a_{2}$. On $S^{n-1} \times I$, we may assume $\nu^{\prime}$ has no zeros, as can be seen from Figure 2.

Now it is clear that the numbers of self-intersection points of $f$ and $f^{\prime}$ differ by 1 and their normal Euler numbers differ by 2 .

Since $f$ and $f^{\prime}$ are obviously bordant, and any bordism class in $\mathscr{N}_{n}(N)$ is represented by a self-transversal immersion, we prove property (1) by repeating the process from $f$ to $f^{\prime}$ and Theorem 2, (1).

If $f$ has even (odd) number of self-intersection points, then $f$ is bordant to an embedding $g$ with $\chi(f)-\chi(g)=2 \times$ even number ( $2 \times$ odd number). This proves (2).

Property (3) is straightforward since

$$
P(x+y)=P(x)+P(y)+2 x \cdot y
$$

and

$$
w_{1}(x+y) w_{n-1}(x+y)=w_{1}(x) w_{n-1}(x)+w_{1}(y) w_{n-1}(y) .
$$

The proof is complete.

\section{Proof of Theorem 4}

We divide the proof into three steps.

Step 1. First, we notice that the homotopy class $[f] \in[M, N]$ is represented by a self-transversal immersion $g: M \rightarrow K$ (cf. [LP]). Regard $g$ as an immersion of $M$ in $N$; then its normal bundle includes a line bundle, hence has an orientation-reversing automorphism. Thus $2 \chi(g)=0$ (cf. [Li1]), and $\chi(g)=0$. We will prove in Steps 2 and 3 that $g$ is regularly homotopic to a self-transversal immersion $f_{1}$ of $M$ in $N$ with mod 2 number of 
self-intersection points $w_{1}(g)\left(u(g)+w_{n-1}(g)\right)$. Since an immersion regularly homotopic to embeddings must have an even number of self-intersections, and $\chi(g)=0$, Theorem 3 follows immediately from the classification theorem of immersions of $M$ in $N$ (cf. [Li2]).

The aim of Step 2 (for $n>2$ ) and Step 3 (for $n=2$ ) is to construct an immersion $f_{1}: M \rightarrow N$ which is self-transversal and regularly homotopic to $g$, and calculate the number of self-intersection points of $f_{1}$.

Step 2. Suppose $n>2$. Then the multiple points of $g$ consist only of double points whose set $X \subset M$ is the disjoint union of some circles $S_{1}, \ldots, S_{k}$ such that $g\left(S_{2 i-1}\right)=g\left(S_{2 i}\right), i=1, \ldots, j$, and $S_{i} \rightarrow g\left(S_{i}\right)$ is a nontrivial 2-sheet covering if $i>2 j$. By formula (1) and the introduction of [He], we see that the homology class $[X]$ in $H_{1}\left(M, Z_{2}\right)$ represented by $X$ is the Poincaré dual of $u(g)+w_{n-1}(g)$.

Let $\varepsilon_{i}= \pm 1 \quad(\delta= \pm 1)$ so that $\varepsilon_{i}=1 \quad\left(\delta_{i}=1\right)$ iff $S_{i}\left(g\left(S_{i}\right)\right)$ is orientationpreserving in $M$ (in $K$ ). Then it is easy to see that

$$
\begin{gathered}
\delta_{2 i-1}=\delta_{2 i}=\varepsilon_{2 i-1} \varepsilon_{2 i} \quad \text { if } 1 \leq i \leq j, \\
\delta_{i}=-\varepsilon_{i} \text { if } 2 j<i \leq k .
\end{gathered}
$$

Denoting by $\xi$ the orientation bundle of $K$, and $\xi_{0}$ the bundle of nonzero vectors of $\xi$, we define $f_{1}$ on $X$ as follows:

(1) If $1 \leq i \leq j$, then $f_{1}=g$ on $S_{2 i-1}$ and $f_{1}=u \circ g$ on $S_{2 i}$, where $u$ is a smooth section of $\xi$ over $g\left(S_{2 i}\right)$ transversal to the zero section.

(2) If $i>2 j$ and $\delta_{i}=-1$, then there is a smooth map $f_{1}: S_{i} \rightarrow \xi_{0}$ such that $p \circ f_{1}=g$, where $p$ is the projection of $\xi$.

(3) If $i>2 j$ and $\delta_{i}=1$, then there is a smooth map $f_{1}: S_{i} \rightarrow \xi$ with $p \circ f_{1}=g$ such that $f_{1}$ has only one transversal self-intersection point.

Extend $f_{1}$ to an immersion of $M$ in $N$ so that $f_{1}=g$ outside a tubular neighbourhood of $X$, and $p \circ f_{1}=g$ on this neighbourhood. Then $f_{1}$ is regularly homotopic to $g$ in $N$ and has only transversal self-intersection points in $f_{1}(X)$.

Now we calculate

$$
\left\langle w_{1}(g),[X]\right\rangle=\sum_{i=1}^{k}\left\langle w_{1}(g),\left[S_{i}\right]\right\rangle .
$$

Letting $s(1)=0$ and $s(-1)=1$, we have

$$
\begin{array}{r}
\left\langle w_{1}(M),\left[S_{i}\right]\right\rangle=s\left(\varepsilon_{i}\right), \\
\left\langle g^{*} w_{1}(K),\left[S_{i}\right]\right\rangle= \begin{cases}s\left(\delta_{i}\right), & 1 \leq i \leq 2 j, \\
0, & 2 j<i \leq k .\end{cases}
\end{array}
$$

It follows then from

$$
w_{1}(M)+w_{1}(g)=g^{*} w_{1}(K)
$$

that

(1) if $1 \leq i \leq j$,

$$
\left\langle w_{1}(g),\left[S_{2 i-1}\right]+\left[S_{2 i}\right]\right\rangle= \begin{cases}0, & \text { if } \delta_{2 i}=1, \\ 1, & \text { if } \delta_{2 i}=-1 ;\end{cases}
$$

(2) if $2 j<i \leq k$ and $\delta_{i}=-1$,

$$
\left\langle w_{1}(g),\left[S_{i}\right]\right\rangle=0
$$


(3) if $2 j<i \leq k$ and $\delta_{i}=1$,

$$
\left\langle w_{1}(g),\left[S_{i}\right]\right\rangle=1 .
$$

This shows that $\left\langle w_{1}(g),[X]\right\rangle$ is equal to mod 2 number of self-intersection points of $f_{1}$. Since

$$
\left\langle w_{1}(g),[X]\right\rangle=w_{1}(g)\left(u(g)+w_{n-1}(g)\right),
$$

the proof for the case $n>2$ is complete.

Step 3. Suppose $n=2$. Now, the multiple points of $g$ consist of double points and triple points whose set $X \subset M$ is the image of a self-transversal immersion $h$ of the disjoint union of some $l$ copies of the circle $S$. Denote by $h_{i}$ the restriction of $h$ on the $i$ th copy of $S$.

Let

$$
X_{1}=\bigcup_{i=1}^{k} h_{i}(S), \quad X_{2}=\bigcup_{i=k+1}^{l} h_{i}(S)
$$

such that each $h_{i}(S)$ in $X_{1}$ includes triple points, while $X_{2}$ does not. Then $X_{1} \cap X_{2}=\varnothing$ and $h_{\alpha}(S) \cap h_{\beta}(S)=\varnothing$, if $k<\alpha<\beta \leq l$, and $X=X_{1} \cup X_{2}$. Moreover, we have

$$
g\left(X_{1}\right) \cap g\left(X_{2}\right)=\varnothing .
$$

We are able to cope with $X_{2}$ exactly as in Step 2. To cope with $X_{1}$, we may assume first that

$$
\begin{gathered}
g\left(h_{2 i-1}(S)\right)=g\left(h_{2 i}(S)\right), \quad \text { if } 1 \leq i \leq j, \\
g\left(h_{i}(S)\right) \neq g\left(h_{\alpha}(S)\right), \quad \text { if } 2 j<i \leq k, \alpha \neq i, 1 \leq \alpha \leq k .
\end{gathered}
$$

We have also

$$
\begin{gathered}
\delta_{2 i-1}=\delta_{2 j}=\varepsilon_{2 i-1} \varepsilon_{2 i}, \quad \text { if } 1 \leq i \leq j, \\
\delta_{i}=-\varepsilon_{i}, \quad \text { if } 2 j<i \leq k,
\end{gathered}
$$

where $\varepsilon_{j}= \pm 1, \delta_{i}= \pm 1$, and $\varepsilon_{i}=1 \quad\left(\delta_{i}=1\right)$ iff $h_{i}: S \rightarrow M \quad\left(g \circ h_{i}: S \rightarrow K\right)$ is an orientation-preserving loop.

Let $X_{3}=\left\{d_{1}, d_{2}, \ldots, d_{3 s}\right\} \subset X_{1}$ be the set of triple points of $g$ which is the set of self-intersection points on $h$ such that

$$
g\left(d_{3 i-2}\right)=g\left(d_{3 i-1}\right)=g\left(d_{3 i}\right), \quad 1 \leq i \leq s,
$$

and let $u$ be a nonzero section of $\xi$ over $g\left(X_{3}\right)$. Let $t: X_{3} \rightarrow R$ be given by

$$
t\left(d_{3 i-2}\right)=-1, \quad t\left(d_{3 i-1}\right)=0, \quad t\left(d_{3 i}\right)=1 .
$$

Step 3(a). Suppose $j \geq 1$ and

$$
h_{1}^{-1}\left(X_{3}\right)=\left\{a_{1}, a_{2}, \ldots, a_{\alpha}\right\} \text {. }
$$

Then

$$
h_{2}^{-1}\left(X_{3}\right)=\left\{b_{1}, b_{2}, \ldots, b_{\alpha}\right\}
$$

has the following properties.

(1) $g\left(h_{1}\left(a_{i}\right)\right)=g\left(h_{2}\left(b_{i}\right)\right)$,

(2) $h_{1}\left(a_{i}\right) \neq h_{2}\left(b_{i}\right)$. 
Regarding $S$ as $[0,1] /\{0,1\}$, we may assume that

$$
\begin{gathered}
a_{1}=0<a_{2}<\cdots<a_{\alpha}<1=a_{\alpha+1}, \\
a_{i}=b_{i}, \quad i=1,2, \ldots, \alpha+1, \\
g\left(h_{1}(q)\right)=g\left(h_{2}(q)\right), \quad \text { for } q \in[0,1] .
\end{gathered}
$$

Define $f_{1}$ on $X_{3}$ by

$$
f_{1}(x)=t(x) u(g(x))
$$

then extend $f_{1}$ to a smooth map on $h_{1}(S)$ so that

$$
f_{1}\left(h_{1}(q)\right)=t_{i}\left(h_{1}(q)\right) u_{i}\left(g\left(h_{1}(q)\right)\right), \quad \text { if } q \in\left[a_{i}, a_{i+1}\right],
$$

where $u_{i}$ is a nonzero smooth section of $\xi$ over $g\left(h_{1}\left(\left[a_{i}, a_{i+1}\right]\right)\right)$ with $\left(u_{i} \circ g \circ h_{1}\right)(q)=\left(u \circ g \circ h_{1}\right)(q)$ for $q=a_{i}, a_{i+1}$, and $t_{i} \circ h_{1}$ is a smooth function on $\left[a_{i}, a_{i+1}\right]$ such that

$$
t_{i}\left(h_{1}(q)\right) u_{1}\left(g\left(h_{1}(q)\right)\right)=t\left(h_{1}(q)\right) u_{1}\left(g\left(h_{1}(q)\right)\right) \text {, for } q=a_{i}, a_{i+1},
$$

and

$$
\frac{d}{d q} t_{i}\left(h_{1}(q)\right)\left\{\begin{array}{l}
=0 \text { on }\left[a_{i}, a_{i}+\varepsilon\right] \cup\left[a_{i+1}-\varepsilon, a_{i+1}\right], \\
\text { is either identically zero or nonzero on }\left(a_{i}+\varepsilon, a_{i+1}-\varepsilon\right),
\end{array}\right.
$$

where $0<\varepsilon<\frac{1}{2}\left(a_{i+1}-a_{i}\right)$ for $i=1,2, \ldots, \alpha$.

Let $v(q)$ be a vector in the fiber of $\xi$ over $g\left(h_{1}(q)\right)$ such that

$$
\begin{aligned}
v\left(a_{i}\right) & =\left(u_{1} \circ g \circ h_{1}\right)\left(a_{1}\right), \\
v(q) & = \pm\left(u_{i} \circ g \circ h_{1}\right)(q), \quad \text { if } q \in\left[a_{i}, a_{i+1}\right],
\end{aligned}
$$

and $v$ as a map $\left[a_{1}, a_{\alpha+1}\right]=[0,1] \rightarrow N$ is continuous. Then $v$ is smooth,

$$
v\left(a_{\alpha+1}\right)=\delta_{1} v\left(a_{1}\right)
$$

and

$$
f_{1}\left(h_{1}(a)\right)=s_{1}(q) v(q),
$$

where $s_{1}$ is a smooth real-valued function on $[0,1]$ such that

$$
\frac{d s_{1}}{d q}\left\{\begin{array}{l}
=0 \text { if }\left|q-a_{i}\right| \leq \varepsilon \text { for some } i, \\
\text { is either identically zero or nonzero on }\left(a_{i}+\varepsilon, a_{i+1}-\varepsilon\right) .
\end{array}\right.
$$

Similarly, we can define

$$
f_{1}\left(h_{2}(q)\right)=s_{2}(q) v(q), \quad q \in[0,1],
$$

with the same $v$, and $s_{2}$ sharing the same properties of $s_{1}$ stated above. Since

$$
f_{1}\left(h_{i}(q)\right)=g\left(h_{i}(q)\right), \quad \text { for } q=0,1 \text { and } i=1,2,
$$

we have

$$
s_{i}(0)=\delta_{i} s_{i}(1), \quad i=1,2 .
$$

Moreover, $s_{1}\left(a_{i}\right)$ and $s_{2}\left(a_{i}\right)$ belong to the set $\{0, \pm 1\}$ and

$$
s_{1}\left(a_{i}\right) \neq s_{2}\left(a_{i}\right), \quad i=1,2, \ldots, \alpha+1,
$$

because $f_{1}\left(h_{1}\left(a_{i}\right)\right) \neq f_{1}\left(h_{2}\left(a_{i}\right)\right)$. Therefore, the graphs of $s_{1}$ and $s_{2}$ intersect transversally, and the number of their intersections is even iff $\delta_{1}=1$. Using the same method, we define $f_{1}$ on $h_{2 i-1}(S) \cup h_{2 i}(S)$ for any $i \in[2, j]$. 
Step 3(b). Assume $k>2 j$ and

$$
h_{k}^{-1}\left(X_{3}\right)=\left\{a_{1}, \ldots, a_{\alpha}, b_{1}, \ldots, b_{\alpha}\right\}
$$

so that $g\left(h_{k}\left(a_{i}\right)\right)=g\left(h_{k}\left(b_{i}\right)\right)$, and $a_{2}, \ldots, a_{\alpha}$ are located in a half-circle bounded by $a_{1}$ and $b_{1}$. Let $p_{1}$ and $p_{2}$ be diffeomorphisms of $[0,1]$ onto the half-circles containing $a_{2}$ and $b_{2}$ respectively such that

$$
g \circ h_{k} \circ p_{1}=g \circ h_{k} \circ p_{2}
$$

and $f \circ h_{k} \circ p_{i}$ is smooth as a map defined on $[0,1] /\{0,1\}$. Then there are a map $v:[0,1] \rightarrow \xi$ and real-valued functions $s_{1}$ and $s_{2}$ on $[0,1]$ as in step $3(\mathrm{a})$, and

$$
\begin{aligned}
v(1) & =\delta_{k} v(0), \\
\left(f_{1} \circ h_{k} \circ p_{i}\right)(q) & =s_{i}(q) v(q), \text { for } q \in[0,1] \text { and } i=1,2, \\
\left(f_{1} \circ h_{k} \circ p_{1}\right)(q) & \neq\left(f_{1} \circ h_{k} \circ p_{2}\right)(q), \quad q \in\{0,1\}, \\
\left(f_{1} \circ h_{k} \circ p_{i}\right)(0) & \neq\left(f_{1} \circ h_{k} \circ p_{i}\right)(1), \quad i=1,2, \\
\left(f_{1} \circ h_{k} \circ p_{1}\right)(0) & =\left(f_{1} \circ h_{k} \circ p_{2}\right)(1), \\
\left(f_{1} \circ h_{k} \circ p_{1}\right)(1) & =\left(f_{1} \circ h_{k} \circ p_{2}\right)(0) .
\end{aligned}
$$

Therefore, the graphs of $s_{1}$ and $s_{2}$ are transversal and the number of their intersections is even iff $\delta_{k}=-1$. Do the same for $h_{i}(S)$ with $2 j<i \leq k$.

Combining Steps 2, 3(a), and 3(b), we have defined $f_{1}$ on $X=X_{1} \cup X_{2} . f_{1}$ can be extended to an immersion of $M$ in $N$ regularly homotopic to $g$ with transversal self-intersection points whose mod 2 number contributed by $X_{1}$ is

$$
\sum_{i=1}^{j} s\left(\delta_{2 i}\right)+\sum_{i=2 j+1}^{k} s\left(-\delta_{i}\right)=\left\langle w_{1}(g),\left[X_{1}\right]\right\rangle .
$$

This together with Steps 1 and 2 proves Theorem 4.

\section{REFERENCES}

[Ha] A. Haefliger, Plongements différentiables de variétés dans variétiés, Comment. Math. Helv. 36 (1961), 47-82.

[He] R. J. Herbert, Multiple points of immersed manifolds, Mem. Amer. Math. Soc., No. 250, 1981.

[Lil] B. H. Li, On reflection of codimension 2 immersions in Euclidean spaces, Sci. Sinica (Ser. A) 31 (1988), 798-805.

[Li2] - On immersions of manifolds in manifolds, Sci. Sinica 25 (1982), 255-263.

[Li3] __ Embeddings of surfaces in 4-manifolds. I, II, Chinese Sci. Bull. 36 (1991), 2025-2033.

[LP] B. H. Li and F. P. Peterson, On immersions of $k$-manifolds in $(2 k-1)$-manifolds, Proc. Amer. Math. Soc. 83 (1981), 159-162.

[Mah] M. Mahowald, On the normal bundle of a manifold, Pacific J. Math. 14 (1964), 1335-1341.

[Mal] B. D. Malyi, The Whitney-Mahowald theorem on normal numbers of smooth embeddings, Mat. Zametki 5 (1969), 91-97. (Russian)

[Mas] W. S. Massey, Pontryagin squares in the Thom space of a bundle, Pacific J. Math. 31 (1969), 133-142.

[Mi] J. Milnor, A procedure for killing the homotopy groups of differentiable manifolds, Proc. Sympos. Pure Math., vol. 3, Amer. Math. Soc., Providence, RI, 1961, pp. 39-55.

[MT] R. E. Mosher and M. C. Tangora, Cohomology operations and applications in homotopy theory, Harper \& Row, New York, Evanston, and London, 1968. 
[Ro] V. A. Rohlin, Proof of Gudkov's hypothesis, Functional Anal. 6 (1972), 136-138.

[Th] E. Thomas, On the cohomology of the real Grassmann complexes and the characteristic classes of n-plane bundles, Trans. Amer. Math. Soc. 96 (1960), 67-89.

[Wh1] H. Whitney, The self-intersection of a smooth n-manifold in 2n-spaces, Ann. of Math. (2) 45 (1944), 220-246.

[Wh2] _ On the topology of differentiable manifolds, Lectures in Topology, Univ. of Michigan Press, 1940.

[Wu] W. T. Wu, On Pontryagin classes. III, Acta Math. Sinica 4 (1954), 323-347.

[Ya] Ya Yamada, An extension of Whitney's congruence, Preprint Series of Univ. of Tokyo, June $15,1993$.

Institute of Systems Science, Academia Sinica, Beijing 100080, China

E-mail address: libh@bepc2. ihep.ac.cn 